Research Paper

\title{
Distinct Patterns of Brain Atrophy associated with Mild Behavioral Impairment in Cognitively Normal Elderly Adults
}

\author{
Jun Shu, MD ${ }^{*}$; Qiang Qiang, MD, Ph.D. ${ }^{*}$; Yuning Yan, MD ${ }^{1}$; Yang Wen, MD²; Yiqing Ren, MD ${ }^{1}$; Wenshi \\ Wei, MD, Ph.D. ${ }^{\boxplus} ;$ Li Zhang, MD, Ph.D. $1^{\square}$ \\ 1. Department of Neurology, Huadong Hospital affiliated to Fudan University, No. 221, West Yan An Road, Shanghai, China. \\ 2. Department of Neurology, The Third People's Hospital of Chengdu, China. \\ *These authors contributed equally to this work. \\ $\square$ Corresponding authors: Wenshi Wei, Department of Neurology, Huadong Hospital affiliated to Fudan University, No. 221, West Yan An Road, Shanghai, \\ China. E-mail: wenshiwei1999@163.com; Phone: +86-13818396027; Li Zhang, Department of Neurology, Huadong Hospital affiliated to Fudan University, No. \\ 221, West Yan An Road, Shanghai, China. E-mail: lizhang_huadong@163.com; Phone: +86-13817955481. \\ (c) The author(s). This is an open access article distributed under the terms of the Creative Commons Attribution License (https://creativecommons.org/licenses/by/4.0/). \\ See http://ivyspring.com/terms for full terms and conditions.
}

Received: 2021.03.24; Accepted: 2021.05.30; Published: 2021.06.11

\begin{abstract}
A cross-sectional study was conducted to evaluate patterns of gray matter changes in cognitively normal elderly adults with mild behavioral impairment (MBI). Sixteen $\mathrm{MBI}$ patients and 18 healthy controls were selected. All the participants underwent a neuropsychological assessment battery, including the Mini-mental State Examination (MMSE), Geriatric Depression Scale (GDS), Self-rating Anxiety Scale (SAS), and Chinese version of the mild behavioral impairment-checklist scale (MBI-C), and magnetic resonance imaging (MRI) scans. Imaging data was analyzed based on voxel-based morphometry (VBM). There was no significant difference in age, gender, MMSE score, total intracranial volume, white matter hyperdensity, gray matter volume, white matter volume between the two groups $(p>0.05)$. MBI group had shorter education years and higher MBI-C score, GDS and SAS scores than the normal control group $(p<0.05)$. For neuroimaging analysis, compared to the normal control group, the MBI group showed decreased volume in the left brainstem, right temporal transverse gyrus, left superior temporal gyrus, left inferior temporal gyrus, left middle temporal gyrus, right occipital pole, right thalamus, left precentral gyrus and left middle frontal gyrus(uncorrected $p<0.001$ ). The grey matter regions correlated with the $\mathrm{MBI}-\mathrm{C}$ score included the left postcentral gyrus, right exterior cerebellum, and left superior frontal gyrus. This suggests a link between $\mathrm{MBI}$ and decreased grey matter volume in cognitively normal elderly adults. Atrophy in the left frontal cortex and right thalamus in MBI patients is in line with frontal-subcortical circuit deficits, which have been linked to neuropsychiatric symptoms (NPS) in dementia. These initial results imply that $\mathrm{MBI}$ might be an early harbinger for subsequent cognitive decline and dementia.
\end{abstract}

Key words: mild behavioral impairment, magnetic resonance imaging, voxel-based morphometry

\section{Introduction}

Neuropsychiatric symptoms (NPS) are standard non-cognitive features of dementia. NPS in dementia can occur at any stage in the disease and are associated with accelerating the rate of cognitive decline [1] and more significant caregiver burden [2]. There is an increasing recognition that NPS are also present in elderly adults with normal cognitive function, known as mild behavioral impairment (MBI) [3]. A growing body of evidence shows that
MBI in cognitively normal older adults can accelerate the decline of their cognitive function and progression to mild cognitive impairment (MCI) and dementia alone or along with MCI [4-7]. Two five-year longitudinal follow-up studies reported that about $70 \%$ of patients with MBI eventually progressed to dementia and MBI patients with MCI were more likely to develop Alzheimer's dementia (AD), whereas MBI patients without MCI were more likely 
to convert into frontotemporal dementia(FTD) $[4,8]$. Lately, some biomarkers of neurodegenerative disease including neurofilament light [9], tau [10], and amyloid [11]; and genetic risk for $\mathrm{AD}$ [12] in cognitively normal samples were found to associate with MBI. A recent study found that MBI scores were associated with global and striatal amyloid-PET signal, but not with tau-PET signal [11]. Conversely, another study showed that MBI scores were associated with higher tau-PET signal in the entorhinal cortex/hippocampus [10]. This discrepancy could be attributed to diverse populations. MBI in elderly adults may be considered a potentially early harbinger of cognitive decline and dementia. Thus, it is crucial to capture the MBI population at high risk for cognitive decline and dementia with the hypothesis that early identification and treatment of behavioral symptoms in neurodegenerative diseases may delay dementia onset or slow its course [3].

To characterize MBI, the MBI Checklist (MBI-C), a screening scale designed to elicit emergent NPS according to the MBI criteria, was developed as a validated and efficient MBI case ascertainment tool [13]. Moreover, the previous two studies have determined cutoff points of the MBI-C in detecting MBI in individuals with MCI [14] and subjective cognitive decline (SCD) [15]. Cui $\mathrm{Y}$ et al. [16] demonstrated that the Chinese version of MBI-C has high reliability and validity and could replace the NPI-Q for AD dementia screening in the Chinese population.

Voxel-based morphometry (VBM) is known as a neuroimaging technique for detecting local changes in brain structure. There is also evidence that VBM can assist in AD diagnosis and early identification of highrisk $\mathrm{MCI}$ population [17]. However, no study to date has investigated the changes of brain structure in MBI patients. The purpose of this study was to determine whether there have been anatomical structural changes of the brain in cognitively normal older adults with MBI (the MBI group) compared to those without MBI (the normal control group) using the VBM technique.

In this study, we employed MBI-C to evaluate NPS and used clinical quality magnetic resonance imaging (MRI) that also could detect changes of regional brain volume reported by Nowrangi MA et al al. [18] to assess regional gray matter volume. A substantial body of literature has shown that NPS's presence in dementia is associated with the structure and function impairment of some distinct brain regions such as the insula, cingulate, prefrontal cortex, striatum, and amygdala [19-21]. Therefore, we posed the hypothesis that there might be some anatomical structural changes of the brain in MBI patients. These brain regions of structural changes may be in line with those related to NPS in dementia.

\section{Methods}

\section{Participants and study design}

We conducted a cross-sectional study involving thirty four cognitively normal older participants aged $\geq 50$ years from inpatient neurology at the Huadong Hospital, Shanghai, China (from August 2018 to August 2019). All participants received clinical, neurological, neuropsychological, and brain MRI examinations. Patients' clinical data (e.g., age, sex, education, hematology test) and neuroimaging data were collected. All participants met the following Mini-mental State Examination (MMSE) scores for education level: 1) middle school level and above $>24$ points; 2) primary school level (education years $>6)>20$ points; 3 ) illiterate $>17$ points. Patients with the presence of acute cerebrovascular disorders, or history of other neurological disorders (e.g., epilepsy, brain tumor, normal pressure hydrocephalus, progressive nuclear palsy, multiple sclerosis), any systemic disease that can cause mental and behavioral symptoms (e.g., vitamin B12 deficiency, hepatic encephalopathy, hypothyroidism), psychiatric illness (e.g., schizophrenia, bipolar disorder, major depression), alcohol or drug abuse or addiction, or diagnosed with Alzheimer's disease or frontotemporal dementia or Lewy body dementia and other neurodegenerative diseases were excluded. A total of 16 patients who met the ISTAART-AA MBI criteria [3] and MBI-C score $>8$ were included in the MBI group, and 18 patients who met MBI-C $=0$ were included in the control group.

This study was approved by the ethics committee of Huadong Hospital (2019K145), and informed consent was obtained for all participants.

\section{Neuropsychological assessment}

All the participants completed a neuropsychological assessment battery, including the MMSE, Geriatric Depression Scale (GDS), Self-rating Anxiety Scale (SAS), and Chinese version of MBI-C. The MBI-C [13], a 2-page questionnaire consisting of 34 items, was structured to be consistent with the five MBI criteria domains: (1) decreased drive/motivation: 6 questions including assessments of cognitive, behavioral and emotional apathy; (2) affective/ emotional dysregulation: 6 items including low mood, anhedonia, hopelessness, and guilt, and 1 question each for worry and panic; (3) impulse dyscontrol: 12 questions assessing agitation, aggression, impulsivity, recklessness, and abnormal reward, and reinforcement; (4) social inappropriateness: 5 
questions describing sensitivity, empathy, and tact; (5) abnormal thoughts/perception: 5 questions assessing suspiciousness, grandiosity, and auditory and visual hallucinations. For each item, a "yes" or "no" question is followed by a severity rating scale of 1, 2, 3, respectively mild, moderate, severe. Symptoms should be persistent for no less than six months and represent a meaningful change from baseline.

\section{MRI acquisition and preprocessing}

High-resolution structural MRI data of the brain were acquired for each patient with MAGNETON Skyra3.0T at the Department of Radiology, Huadong Hospital, Shanghai. MRI was performed on all subjects, including horizontal T1WI and T2WI, diffusion-weighted imaging (DWI), fluid-attenuated inversion recovery (FLAIR). The scanning parameters were horizontal T1WI: repetition time/echo time $(\mathrm{TR} / \mathrm{TE})=220 / 2.46 \mathrm{~ms}$, slice thickness $=5 \mathrm{~mm}$, spacing $=1.0 \mathrm{~mm}, \mathrm{FOV}=23.00 \mathrm{~cm}$; T2WI: TR $/ \mathrm{TE}=$ $4000 / 92 \mathrm{~ms}$, slice thickness $=5 \mathrm{~mm}, \mathrm{FOV}=24.00 \mathrm{~cm}$; FLAIR: TR/TE $=7000 / 85 \mathrm{~ms}$, slice thickness $=5 \mathrm{~mm}$, FOV $=23.00 \mathrm{~cm}$; DWI: TR $/ \mathrm{TE}=1300 / 62 \mathrm{~ms}$, slice thickness $=5 \mathrm{~mm}, \mathrm{FOV}=24.00 \mathrm{~cm}$. Structural MRI images were preprocessed using the CAT12 (http:/ / www.neuro.uni-jena.de/cat/) toolbox of the SPM12 (Statistical Parametric Mapping, V.12b; http://www. fil.ion.ucl.ac.uk/spm) running in Matlab V.R2014b (uk.mathworks.com/products/matlab/). Firstly, the primary DICOM format of T1MRI images of all subjects were converted into NIFTI format files by the DICOM import function of spm12. Secondly, the converted NIFTI images were segmented into the gray matter (GM), white matter (WM), and cerebrospinal fluid (CSF). Thirdly, the gray matter was spatially normalized and modulated in DARTEL (Diffeomorphic Anatomical Registration through Exponentiated Lie Algebra) method [22] and registered to Montreal Neurological Institute (MNI) space. The modulated and normalized but unsmoothed GM images were visually inspected for artifacts using the sample homogeneity module. Those participants whose GM volume was two or more standard deviations outside of the GM volume sample distributions center would be excluded. No participant was excluded in this study. Lastly, $8 \mathrm{~mm}$ full-width at half maximum (FWHM) isotropic Gaussian kernel was adopted to smooth the above preprocessed GM image. The smoothed datasets were prepared for statistical analysis.

\section{Statistical analysis}

The demographical and clinical data analysis was completed with SPSS 23.0. All the continuous variables were shown by mean \pm standard deviation, and the constituent ratio described gender. Normality tests were performed by the Shapiro-Wilk test because the sample size of our study was less than 50 . Two independent sample t-test was applied when samples satisfied the standard normal distribution. Two independent samples non-parametric test (Mann-Whitney $U$ test) was done when samples did not meet a standard normal distribution. Fisher's exact test was conducted to compare the gender difference between the two groups because of the small sample size $(<40)$.

For image analysis, the smoothed datasets from the above preprocessed GM image were analyzed using a linear regression analysis to compare the differences of grey matter volume between the MBI group and the control group and to explore gray matter changes correlated to the MBI-C score in the MBI group. Age, years of education, sex, and total intracranial volume (TIV) were included as covariates. Significant effects were determined at the threshold of uncorrected $p<0.001$ and with cluster size above 20 voxels. The image analysis was using SPM12 software.

\section{Results}

\section{Clinical characteristics of patients}

The demographic characteristics of the MBI group and normal control group are displayed in Table 1. There were no significant differences between the MBI group and the controls concerning age, gender, MMSE score, TIV, white matter hyperdensity, gray matter volume, white matter volume $(p>0.05)$. Significant group differences were founded in education years, MBI-C score, GDS, and SAS scores between the two groups $(p<0.05)$. Compared to the normal control group, the MBI group had shorter education years and higher MBI-C score, GDS, and SAS scores.

\section{Comparison of grey matter volume between the MBI group and control group}

The MBI group showed gray matter atrophy in the left brainstem, right temporal transverse gyrus, left superior temporal gyrus, left inferior temporal gyrus, left middle temporal gyrus, right occipital pole, right thalamus, left precentral gyrus and left middle frontal gyrus (uncorrected $p<0.001$ ) compared to the normal control group (Fig. 1 and Table 2).

\section{Grey matter volume changes related to $\mathrm{MBI}-\mathrm{C}$ score}

We further explored the gray matter volume changes correlated to the MBI-C score and found the grey matter regions correlated with the MBI-C score included left postcentral gyrus, right exterior 
cerebellum, and left superior frontal gyrus (uncorrected $p<0.001$ ) (Fig. 2 and Table 3).

Table 1. Demographic data of the participants

\begin{tabular}{llll}
\hline & MBI group & Control group & P value \\
\hline Total number (n) & 16 & 18 & \\
Gender (man/female) & $6 / 10$ & $10 / 8$ & $0.327^{\mathrm{a}}$ \\
Age (years) & $67.31 \pm 6.69$ & $66.67 \pm 7.18$ & $0.789^{\mathrm{b}}$ \\
Education (years) & $9.31 \pm 1.54$ & $11.6 \pm 3.12$ & $0.025^{*_{\mathrm{c}}}$ \\
MMSE score & $28.19 \pm 1.22$ & $28.78 \pm 0.81$ & $0.103^{\mathrm{b}}$ \\
GDS score & $10.19 \pm 3.78$ & $2.67 \pm 1.50$ & $0.000^{*_{\mathrm{c}}}$ \\
SAS score & $51.19 \pm 4.87$ & $28.39 \pm 2.73$ & $0.000^{\star_{\mathrm{c}}}$ \\
MBI-C total score & $10.38 \pm 1.45$ & $0.00 \pm 0.00$ & $0.000^{\star_{\mathrm{c}}}$ \\
decreased drive/motivation & $3.56 \pm 1.71$ & $0.00 \pm 0.00$ & - \\
affective/emotional dysregulation & $5.12 \pm 1.54$ & $0.00 \pm 0.00$ & - \\
impulse dyscontrol & $1.81 \pm 1.68$ & $0.00 \pm 0.00$ & - \\
social inappropriateness & $0.00 \pm 0.00$ & $0.00 \pm 0.00$ & - \\
abnormal thoughts/perception & $0.00 \pm 0.00$ & $0.00 \pm 0.00$ & - \\
total intracranial volume $\left(\mathrm{mm}^{3}\right)$ & $1752.61 \pm 72.29$ & $1753.32 \pm 54.90$ & $0.975^{\mathrm{b}}$ \\
gray matter volume $\left(\mathrm{mm}^{3}\right)$ & $665.79 \pm 31.01$ & $672.02 \pm 28.04$ & $0.543^{\mathrm{b}}$ \\
white matter volume $\left(\mathrm{mm}^{3}\right)$ & $665.74 \pm 41.50$ & $661.96 \pm 49.03$ & $0.811^{\mathrm{b}}$ \\
white matter hyperdensity & $13.64 \pm 3.70$ & $13.91 \pm 5.39$ & $0.866^{\mathrm{b}}$ \\
\hline
\end{tabular}

Note: a Fisher's Exact Tests; b two-sample t-test; c nonparametric test (Mann-Whitney $\mathrm{U}$ test). $\mathrm{p}<0.05$ was considered significant, ${ }^{*}$ represents significant difference.

Abbreviations: MMSE, Mini-Mental State Examination; GDS: Geriatric Depression
Scale; SAS: Self-rating Anxiety Scale, MBI-C: mild behavioral impairment checklist.

Table 2. Voxel-based morphometry differences between mild behavioral impairment group $(n=16)$ and control group $(n=18)$

\begin{tabular}{lllllll}
\hline $\begin{array}{l}\text { Cluster } \begin{array}{l}\text { size } \\
\text { (voxel) }\end{array} \\
\text { Peak level }\end{array}$ & $\begin{array}{l}\text { MNI Coordinates } \\
(\mathrm{mm})\end{array}$ & $\begin{array}{l}\mathrm{T} \\
\text { value }\end{array}$ & $\begin{array}{l}\mathrm{P} \\
\text { uncorrected }\end{array}$ \\
\cline { 2 - 5 } & & $\mathrm{x}$ & $\mathrm{y}$ & $\mathrm{z}$ & & \\
\hline 68 & left brainstem & -11 & -38 & -60 & 4.56 & 0.000 \\
130 & right temporal transverse gyrus & 42 & -24 & 6 & 4.54 & 0.000 \\
65 & Right Thalamus & 17 & -14 & -2 & 4.23 & 0.000 \\
40 & left superior temporal gyrus & -45 & 0 & -21 & 4.19 & 0.000 \\
54 & left inferior temporal gyrus & -45 & -41 & -21 & 4.16 & 0.000 \\
89 & right occipital pole & 3 & -95 & 8 & 4.14 & 0.000 \\
23 & left precentral gyrus & -65 & 8 & 12 & 3.97 & 0.000 \\
56 & left middle temporal gyrus & -53 & -62 & 5 & 3.81 & 0.000 \\
29 & left middle frontal gyrus & -32 & 35 & 20 & 3.77 & 0.000 \\
\hline
\end{tabular}

\section{Discussion}

To our knowledge, this was the first study to investigate patterns of gray matter changes in MBI patients based on VBM. In addition, the MBI-C was used to capture MBI in our study, which is specifically
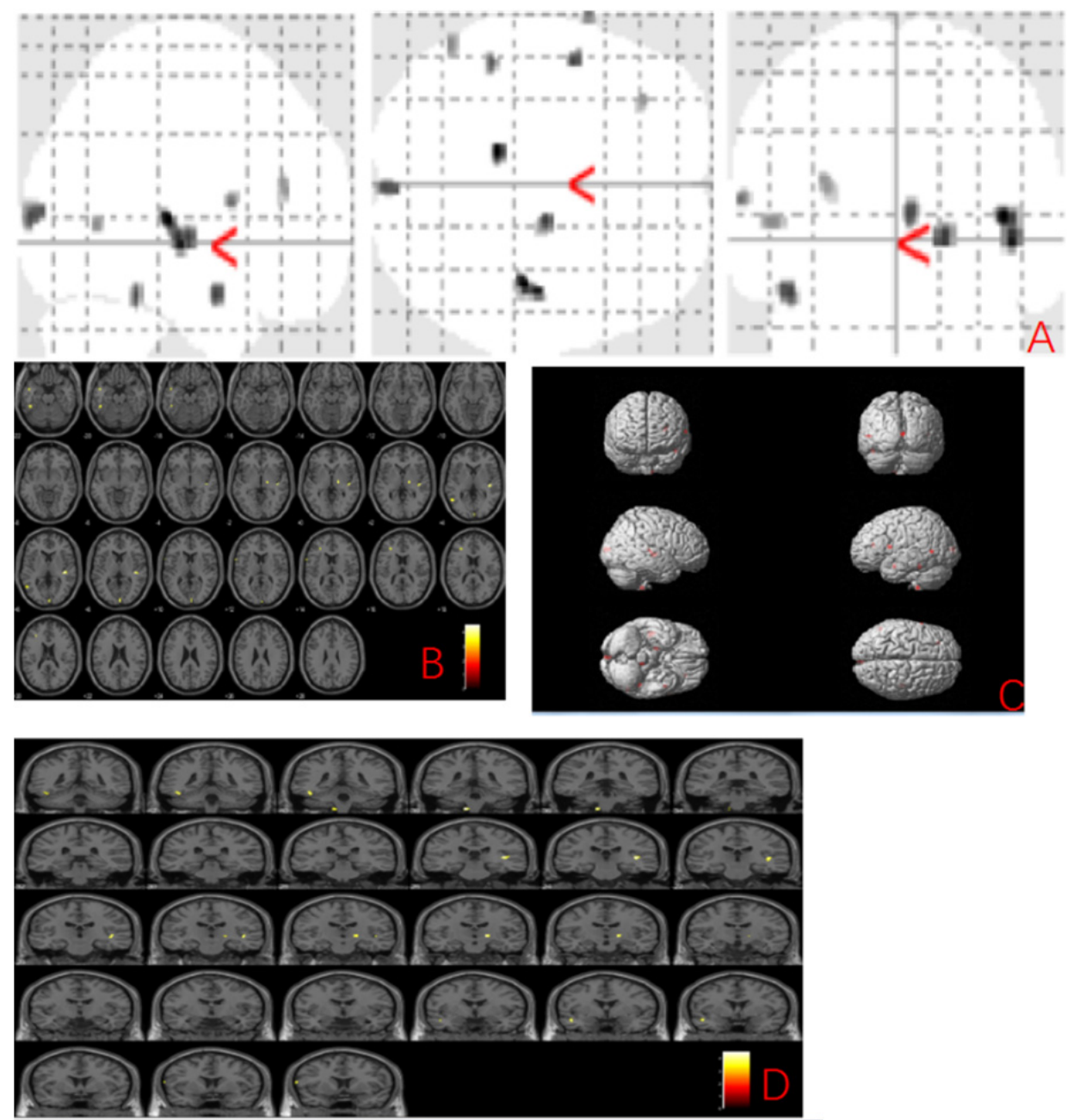

Figure 1. Anatomic location of brain regions showing significant gray matter volume decrease in the mild behavioral impairment group compared to the control group. Upper panel: Maximum density transparency graphs (A), where gray matter volume reduction was labeled with grey color. Lower panel: Horizontal (B), coronal (D) brain graphs, where gray matter volume reduction was labeled with yellow color; 3-D brain surface rendering graphs (C), where gray matter volume reduction was labeled with red color. 
designed for assessing MBI [13]. The MBI-C is a more precise approach tool than the NPI or other nonspecific instruments. This study showed that the MBI group had a shorter education year and higher MBI-C score, GDS and SAS scores than the normal control group. There was no difference between the MBI group and the normal control on age, gender, MMSE score, TIV, white matter hyperdensity, gray matter volume, and white matter volume. For neuroimaging analyses, we observed that MBI patients had significant decreased gray matter volume in the left brainstem, bilateral temporal cortex (mainly on the left), right occipital pole, right thalamus, left frontal cortex (uncorrected $p<0.001$ ) compared to the normal control group after adjusting for TIV, age, gender, and years of education. The finding indicates associations between MBI and anatomical structural changes of the brain, which might provide insight into the anatomical basis of MBI in older adults.

Table 3. Anatomic location of areas related to $\mathrm{MBI}-\mathrm{C}$ score

\begin{tabular}{|c|c|c|c|c|c|c|}
\hline \multirow{2}{*}{$\begin{array}{l}\text { Cluster } \\
\text { size (voxel) }\end{array}$} & \multirow[t]{2}{*}{ Peak-level } & \multicolumn{3}{|c|}{ MNI Coordinates (mm) } & \multirow{2}{*}{$\begin{array}{l}F \\
\text { value }\end{array}$} & \multirow{2}{*}{$\begin{array}{l}\mathrm{P} \\
\text { uncorrected }\end{array}$} \\
\hline & & $\mathrm{x}$ & $\mathrm{y}$ & $\mathrm{z}$ & & \\
\hline 147 & left postcentral gyrus & -54 & -18 & 39 & 43.56 & 0.000 \\
\hline 62 & $\begin{array}{l}\text { right exterior } \\
\text { cerebellum }\end{array}$ & 44 & -57 & -26 & 32.36 & 0.000 \\
\hline 36 & $\begin{array}{l}\text { left superior frontal } \\
\text { gyrus }\end{array}$ & -14 & 54 & 32 & 28.26 & 0.000 \\
\hline
\end{tabular}

The frontal cortex and thalamus are part of the frontal-subcortical circuit consisting of the frontal cortex, striatum, globus pallidus, substantia nigra, and thalamus. The circuit originates in the frontal cortex and sequentially projects to the striatum; to the globus pallidus and substantia nigra; then to the specific thalamic nuclei, and finally back to the frontal cortex and integrity of its structure and function can promote organisms to interact adaptively with their environment [23]. This study showed decreased gray matter volume in the left frontal cortex and right thalamus in MBI patients, indicating that the presence of MBI in older adults may be related to the structural integrity impairment of the frontal-subcortical circuit, which was in line with the view that NPS in dementia is closely linked to impaired structural or functional
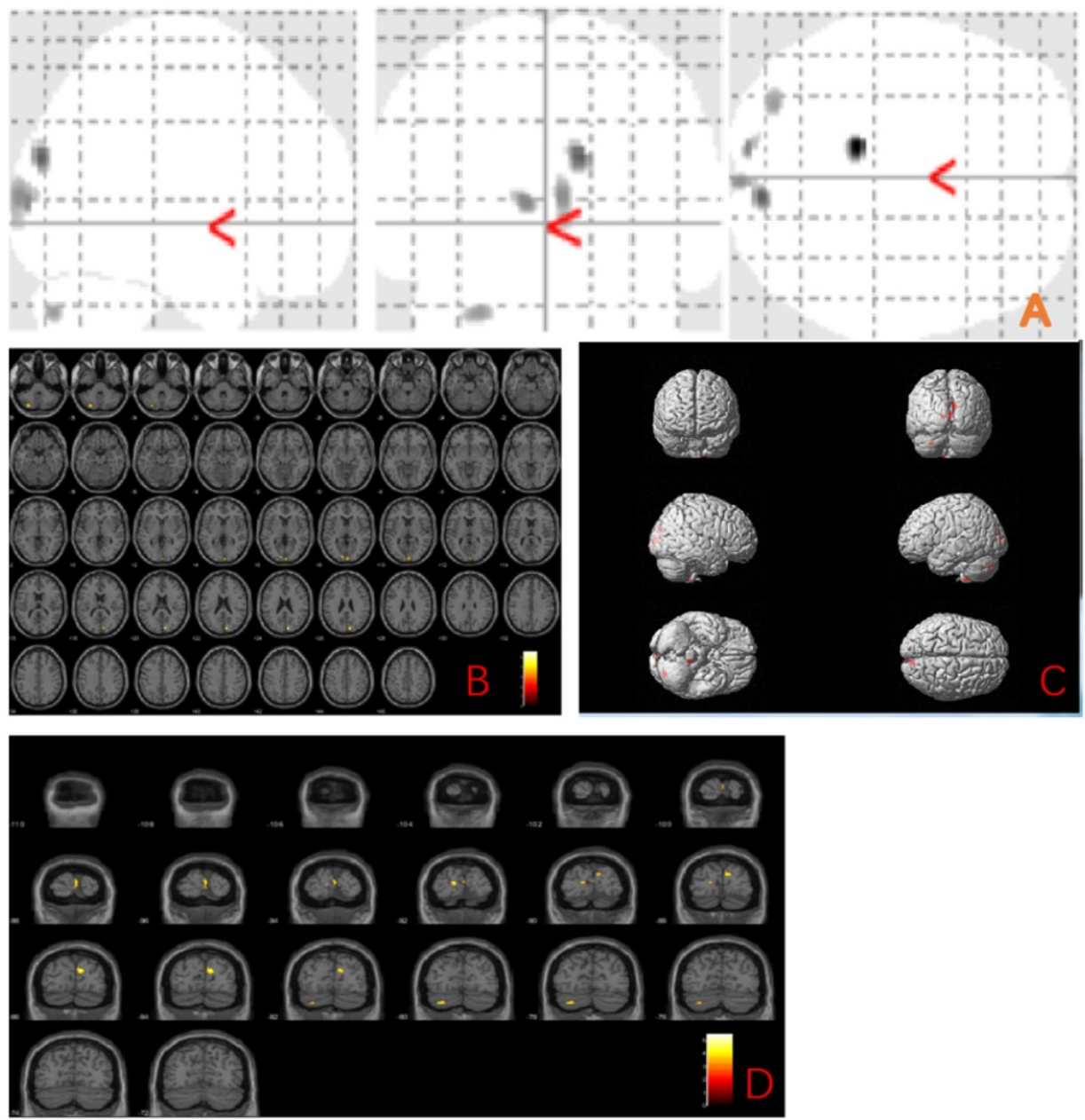

Figure 2. Anatomic location of brain regions showing significant correlations between gray matter atrophy and MBI-C score. Upper panel: Maximum density transparency graphs (A), where gray matter volume reduction was labeled with grey color. Lower panel: Horizontal (B), coronal (D) brain graphs, where gray matter volume reduction was labeled with yellow color; 3-D brain surface rendering graphs (C), where gray matter volume reduction was labeled with red color. 
integrity of frontal-subcortical circuits [24]. Prior studies have consistently reported disturbance of frontal-subcortical circuits in dementia with NPS [19, 21]. AD patients with apathy symptoms had significantly more significant cortical thinning in the left lateral orbitofrontal cortex (OFC), left superior and ventrolateral frontal regions than those without apathy symptoms [25]. A functional MRI study revealed that $\mathrm{MCI}$ patients with greater affective symptoms were related to the decreased connectivity in the frontal-parietal control networks. Our findings, together with prior results, provide new evidence for a continuum between MBI and dementia. Multimodality MRI such as diffusion tensor imaging (DTI) and resting-state functional MRI (fMRI), should be applied to test the hypothesis in the future.

Our study also found gray matter structure changes of MBI patients mainly in the left temporal lobe, which, on the one hand, implied that MBI patients were in an at-risk state for incident cognitive decline and dementia, and on the other hand, supported the association of temporal lobe with NPS. The temporal lobe, especially the medial temporal lobe, is closely related to memory function [26]. There is widespread agreement that the medial temporal lobe atrophy is a core bio-marker of early $\mathrm{AD}$ and is associated with cognitive decline and disease progression in dementia [27, 28]. Patients with amnestic $\mathrm{MCI}$, who converted to $\mathrm{AD}$, showed bilateral gray matter loss in the medial and inferior temporal lobe at baseline compared to those who did not progress to $A D$ [29]. A recent study reported that amnestic MCI and other MCI subtypes such as dysnomic/amnestic MCI and mixed MCI all showed medial temporal lobe thinning compared to healthy elderly adults, while different MCI subtypes demonstrated unique longitudinal atrophy patterns, which may have paramount prognostic value for promoting the prediction of clinical course [30]. Besides, there is some evidence that cortical thinning, tau accumulation and lower metabolism in the temporal lobe was associated with individual NPS such as apathy in both cognitively normal elderly (CN) and MCI [31], depressive symptoms in $\mathrm{CN}$ [32, 33], and anxiety symptoms in AD [34], respectively. These results indicate that MBI patients with temporal cortical atrophy should be closely monitored to determine whether such a population has a higher risk of conversion to $\mathrm{MCI}$ or dementia.

Decreased gray matter volume in the left brainstem of MBI patients was found compared with the normal control group. This difference remained significant after adjusting for sex, age, years of education, and TIV. The reticular structure is the core of the brain stem, which integrates all kinds of signals from the cortex and subcortical regions, and participates in regulating the human sleep-wake state, emotional behavior, temperature, and other functions [35]. Impaired brainstem structural integrity may reduce attention networks and levels of excitement, leading to changes in emotional behavior. However, few studies on the correlation between NPS and brain stem and more studies are needed to further determine the relationship between them.

We further explored the brain regions associated with the MBI-C score and found that gray matter changes associated with the MBI-C score included the left central posterior gyrus, the left superior frontal gyrus, and the right lateral cerebellar gyrus, suggesting that cerebellar structural change was also closely related to NPS. The primary function of the cerebellum is balance and coordination, but there is increasing recognition on the role of the cerebellum in social cognition and emotion. Baillieux et al. [36] showed that small brain damage caused by trauma, tumor, infection, or stroke could cause clinically significant cognitive and affective disturbances. Wassink et al. [37] reported that decreased cerebellar volume was associated with negative psychotic symptoms in schizophrenia patients compared with normal controls. Our findings were in accordance with previous studies tending to support the hypothesis that the cerebellum also plays an essential role in social cognition and emotion.

There are several limitations worthy of discussion. Our study's sample size is relatively small, which may weaken the interpretation of our findings; therefore, future larger population-based cohorts are needed to confirm these results. This study was a cross-sectional study, and we could not evaluate the association between distinct longitudinal atrophy patterns of MBI patients and the disease progression, so longitudinal studies should be designed to elaborate on this issue. When analyzing image data, we did not use multiple comparisons correction. However, the threshold of $p \leq 0.001$ (and not 0.05), as usually used in the VBM study, was set to overcome the chance factor [38].

In conclusion, this study shows that anatomical structural changes were present in the brain of MBI patients and VBM techniques can detect microstructural changes. Decreased gray matter volume of MBI patients in the left frontal cortex and right thalamus is in line with frontal-subcortical circuit deficits, which have been linked to NPS in dementia. The left temporal lobe atrophy was also founded in MBI patients, which is associated with cognitive decline and disease progression in patients with dementia. These initial results imply that MBI might be an early harbinger for subsequent cognitive 
decline and dementia.

\section{Acknowledgements}

This work was supported by Shanghai Municipal Health Commission [grant numbers 2020YJZX0109] and Shanghai Pujiang Program [grant numbers: 2019PJD013]; The funder had no role in study design, data analysis, decision to publish, or preparation of the manuscript. The authors would like to thank Shanghai Municipal Health Commission for their support.

\section{Competing Interests}

The authors have declared that no competing interest exists.

\section{References}

1. Lanctôt KL, Amatniek J, Ancoli-Israel S, et al. Neuropsychiatric signs and symptoms of Alzheimer's disease: New treatment paradigms. Alzheimer's \& Dementia: Translational Research \& Clinical Interventions. 2017; 3(3): 440-49.

2. Lima-Silva TB, Bahia VS, Carvalho VA, et al. Neuropsychiatric Symptoms, Caregiver Burden and Distress in Behavioral-Variant Frontotemporal Dementia and Alzheimer's Disease. Dementia and Geriatric Cognitive Disorders. 2015; 40(5-6): 268-75.

3. Ismail Z, Smith EE, Geda Y, et al. Neuropsychiatric symptoms as early manifestations of emergent dementia: Provisional diagnostic criteria for mild behavioral impairment. Alzheimer's \& Dementia. 2016; 12(2): 195-202.

4. Taragano FE, Allegri RF, Heisecke SL, et al. Risk of Conversion to Dementia in a Mild Behavioral Impairment Group Compared to a Psychiatric Group and to a Mild Cognitive Impairment Group. J Alzheimers Dis. 2018; 62(1): 227-38.

5. Wise EA, Rosenberg PB, Lyketsos CG, et al. Time course of neuropsychiatric symptoms and cognitive diagnosis in National Alzheimer's Coordinating Centers volunteers. Alzheimer's \& Dementia: Diagnosis, Assessment \& Disease Monitoring. 2019; 11: 333-39.

6. Creese B, Brooker H, Ismail Z, et al. Mild Behavioral Impairment as a Marker of Cognitive Decline in Cognitively Normal Older Adults. The American Journal of Geriatric Psychiatry. 2019; 27(8): 823-34.

7. Forrester SN, Gallo JJ, Smith GS, et al. Patterns of Neuropsychiatric Symptoms in Mild Cognitive Impairment and Risk of Dementia. The American Journal of Geriatric Psychiatry. 2016; 24(2): 117-25.

8. Taragano FE, Allegri RF, Krupitzki H, et al. Mild behavioral impairment and risk of dementia: a prospective cohort study of 358 patients. J Clin Psychiatry. 2009; 70(4): 584-92.

9. Naude JP, Gill S, Hu S, et al. Plasma Neurofilament Light: A Marker of Neurodegeneration in Mild Behavioral Impairment. Journal of Alzheimer's Disease. 2020; 76(3): 1017-27.

10. Johansson M, Stomrud E, Insel PS, et al. Mild behavioral impairment and its relation to tau pathology in preclinical Alzheimer's disease. Translational Psychiatry. 2021; 11(1)

11. Lussier FZ, Pascoal TA, Chamoun $\mathrm{M}$, et al. Mild behavioral impairment is associated with $\beta$-amyloid but not tau or neurodegeneration in cognitively intact elderly individuals. Alzheimer's \& dementia. 2020; 16(1): 192-99.

12. Creese B, Arathimos R, Brooker $\mathrm{H}$, et al. Genetic risk for Alzheimer's disease, cognition, and mild behavioral impairment in healthy older adults. Alzheimer's \& Dementia: Diagnosis, Assessment \& Disease Monitoring. 2021; 13(1).

13. Ismail $\mathrm{Z}$, Agüera-Ortiz L, Brodaty $\mathrm{H}$, et al. The Mild Behavioral Impairment Checklist (MBI-C): A Rating Scale for Neuropsychiatric Symptoms in PreDementia Populations. Journal of Alzheimer's Disease. 2017; 56(3): 929-38.

14. Mallo SC, Ismail Z, Pereiro AX, et al. Assessing Mild Behavioral Impairment with the Mild Behavioral Impairment-Checklist in People with Mild Cognitive Impairment. Journal of Alzheimer's Disease. 2018; 66(1): 83-95.

15. Mallo SC, Ismail Z, Pereiro AX, et al. Assessing mild behavioral impairment with the mild behavioral impairment checklist in people with subjective cognitive decline. International Psychogeriatrics. 2018: 1-09.

16. Cui Y, Dai S, Miao Z, et al. Reliability and Validity of the Chinese Version of the Mild Behavioral Impairment Checklist for Screening for Alzheimer's Disease. Journal of Alzheimer's Disease. 2019; 70(3): 747-56.

17. Gupta Y, Lee KH, Choi KY, et al. Early diagnosis of Alzheimer's disease using combined features from voxel-based morphometry and cortical, subcortical, and hippocampus regions of MRI T1 brain images. PLOS ONE. 2019; 14(10): e222446.

18. Nowrangi MA, Marano C, Oishi K, et al. The association of neuropsychiatric symptoms with regional brain volumes from patients in a tertiary multidisciplinary memory clinic. International Psychogeriatrics. 2020: 1-12.
19. Rosenberg PB, Nowrangi MA, Lyketsos CG. Neuropsychiatric symptoms in Alzheimer's disease: What might be associated brain circuits? Molecular Aspects of Medicine. 2015; 43-44: 25-37.

20. Pink A, Przybelski SA, Krell-Roesch J, et al. Cortical Thickness and Anxiety Symptoms among Cognitively Normal Elderly Persons: The Mayo Clinic Study of Aging. The Journal of Neuropsychiatry and Clinical Neurosciences. 2017; 29(1): 60-66.

21. Geda YE, Schneider LS, Gitlin LN, et al. Neuropsychiatric symptoms in Alzheimer's disease: Past progress and anticipation of the future. Alzheimer's \& Dementia. 2013; 9(5): 602-08.

22. Ashburner J. A fast diffeomorphic image registration algorithm. Neuroimage. 2007; 38(1): 95-113.

23. Cummings JL. Frontal-subcortical circuits and human behavior. Archives of neurology. 1993; 50(8): 873

24. Bonelli RM, Cummings JL. Frontal-subcortical circuitry and behavior. Dialogues in clinical neuroscience. 2007; 9(2): 141-51.

25. Tunnard C, Whitehead D, Hurt C, et al. Apathy and cortical atrophy in Alzheimer's disease. Int J Geriatr Psychiatry. 2011; 26(7): 741-48.

26. Lech RK, Suchan B. The medial temporal lobe: memory and beyond. Behav Brain Res. 2013; 254: 45-49.

27. Pini L, Pievani M, Bocchetta M, et al. Brain atrophy in Alzheimer's Disease and aging. Ageing Research Reviews. 2016; 30: 25-48.

28. Frisoni GB, Prestia A, Rasser PE, et al. In vivo mapping of incremental cortical atrophy from incipient to overt Alzheimer's disease. Journal of Neurology. 2009; 256(6): 916-24

29. Whitwell JL, Shiung MM, Przybelski SA, et al. MRI patterns of atrophy associated with progression to $\mathrm{AD}$ in amnestic mild cognitive impairment. Neurology. 2008; 70(7): 512-20.

30. Edmonds EC, Weigand AJ, Hatton SN, et al. Patterns of longitudinal cortical atrophy over 3 years in empirically derived MCI subtypes. Neurology. 2020; 94(24): e2532-44.

31. Guercio BJ, Donovan NJ, Ward A, et al. Apathy is associated with Lower Inferior Temporal Cortical Thickness in Mild Cognitive Impairment and Normal Elderly Individuals. The Journal of Neuropsychiatry and Clinical Neurosciences. 2015; 27(1): e22-27.

32. Pink A, Przybelski SA, Krell-Roesch J, et al. Cortical Thickness and Depressive Symptoms in Cognitively Normal Individuals: The Mayo Clinic Study of Aging. Journal of Alzheimer's Disease. 2017; 58(4): 1273-81.

33. Donovan NJ, Hsu DC, Dagley AS, et al. Depressive Symptoms and Biomarkers of Alzheimer's Disease in Cognitively Normal Older Adults. J Alzheimers Dis. 2015; 46(1): 63-73.

34. Hashimoto H, Monserratt L, Nguyen P, et al. Anxiety and regional cortical glucose metabolism in patients with Alzheimer's disease. J Neuropsychiatry Clin Neurosci. 2006; 18(4): 521-28.

35. Schepens B, Stapley $\mathrm{P}$, Drew $\mathrm{T}$. Neurons in the pontomedullary reticular formation signal posture and movement both as an integrated behavior and independently. J Neurophysiol. 2008; 100(4): 2235-53.

36. Baillieux H, De Smet HJ, Dobbeleir A, et al. Cognitive and affective disturbances following focal cerebellar damage in adults: a neuropsychological and SPECT study. Cortex. 2010; 46(7): 869-79.

37. Wassink TH, Andreasen NC, Nopoulos P, et al. Cerebellar morphology as a predictor of symptom and psychosocial outcome in schizophrenia. Biol Psychiatry. 1999; 45(1): 41-48

38. Shen S, Sterr A. Is DARTEL-based voxel-based morphometry affected by width of smoothing kernel and group size? A study using simulated atrophy. J Magn Reson Imaging. 2013; 37(6): 1468-75. 